\title{
Estoques de carbono orgânico do solo em cafezais sob diferentes sistemas de controle de plantas invasoras
}

\section{Soil organic carbon stocks in coffee plantations under different weed control systems}

\author{
Franciane Diniz Cogo ${ }^{1 *}$; Cezar Francisco Araujo-Junior ${ }^{2}$; \\ Yuri Lopes Zinn ${ }^{3}$; Moacir de Souza Dias Junior"; \\ Elifas Nunes de Alcântara ${ }^{5}$; Paulo Tácito Gontijo Guimarães ${ }^{5}$
}

\section{Resumo}

O carbono orgânico no solo (COS) é um importante indicador da qualidade do solo, pois seus teores e estoques podem ser alterados conforme o sistema de preparo do solo. Objetivou-se neste trabalho avaliar os estoques de COS em um Latossolo Vermelho distroférrico argiloso cultivado com cafeeiros (Coffea arabica L) e submetido a diferentes manejos de plantas invasoras no sul de Minas Gerais. O delineamento experimental foi em blocos ao acaso, sendo os tratamentos os seguintes métodos de controle de plantas invasoras: sem capina, capina manual, herbicida de pré-emergência, herbicida de pós-emergência, enxada rotativa, roçadora e grade. Amostras de solo com estrutura indeformada foram coletadas em duas posições (entrelinha e linha do tráfego), nas profundidades de 0-3, 10-13 e 25-28 $\mathrm{cm}$. Uma mata nativa adjacente foi amostrada como referência. Verificou-se maior densidade do solo na área sob cafezal em comparação ao solo sob a mata. Houve pouca diferença entre o teor de COS da posição entrelinha dos cafeeiros em relação àquele sob mata, mas para a posição linha de tráfego, o teor de COS foi menor. Após correção para a compactação do solo, verificou-se perda de aproximadamente $20 \%$ nos estoque de COS a 0-30 cm quando se utilizou herbicida de pós-emergência, enxada rotativa, capina manual e grade, e perdas de $35 \%$, quando se utilizou herbicida de pré-emergência. Os manejos sem capina e roçadora não diferiram da mata nativa $\left(37 \mathrm{Mg} \mathrm{COS} \mathrm{ha}^{-1}\right)$, indicando que a roçadora, por permitir crescimento temporário das invasoras e não revolver o solo, é o controle mais apropriado à conservação do COS em cafezais.

Palavras-chave: Coffea arabica L., plantas daninhas, matéria orgânica do solo

\footnotetext{
Abstract

Soil organic carbon (SOC) is an important indicator of soil quality, as its levels and stocks can change by soil preparation. This study aimed to evaluate SOC stocks on a clayey Oxisol cultivated with coffee and subject to different weed control systems in southern Minas Gerais, Brazil. The experimental design was in randomized blocks, and weed control systems were: no weeding, manual weeding, pre-emergence herbicide, post-emergence herbicide, rotary tiller, rotary mowers and disk harrow. Undisturbed soil samples were collected at two positions in the coffee plantation (tire tracks and planting line), at depths

${ }^{1}$ Mestranda do Programa de Pós-Graduação em Ciência do Solo, Universidade Federal de Lavras, UFLA, Lavras, MG. E-mail: fdcogo@yahoo.com.br

${ }^{2}$ Pesquisador da Área de Solos do Instituto Agronômico do Paraná, IAPAR, Londrina, PR. E-mail: cezar_araujo@iapar.br

${ }^{3}$ Prof. do Dept ${ }^{\circ}$ de Ciência do Solo da UFLA, Lavras, MG. E-mail: ylzinn@des.ufla.br

${ }^{4}$ Prof. do Dept ${ }^{\circ}$ de Ciência do Solo da UFLA. Bolsista de produtividade CNPQ, Lavras, MG. E-mail: msouzadj@dcs.ufla.br

${ }_{5}^{5}$ Pesquisadores, Empresa de Pesquisa Agropecuária de Minas Gerais, EPAMIG, campus Universidade Federal de Lavras, Lavras, MG. E-mail: elifas@epamig.ufla.br; paulotgg@epamig.ufla.br

*Autor para correspondência
} 
of $0-3,10-13$, and $25-28 \mathrm{~cm}$. A nearby native forest was sampled as a reference. A higher bulk density of soils under coffee plantations occurred compared to soil under the forest. There was little difference between SOC concentrations in the plating line in relation to the native forest, but for the tire track position, the amount of SOC was generally lower. After correction for soil compaction, it was estimated a loss of ca. 20\% in SOC stock for the 0-30 cm depth for herbicide post-emergence, rotary tiller, manual weeding and disk harrow, and a 35\% loss when using herbicide pre-emergence. SOC stocks under no weeding and rotary mowers did not differ from native forest $\left(37 \mathrm{M}^{-1} \mathrm{~g}\right.$ ha SOC), indicating that the rotary mower, which allows temporary growth of weeds and does not disrupt soil structure, is the most appropriate weed control for the preservation of COS in coffee plantations.

Key words: Coffea arabica L., weeds, soil organic matter

\section{Introdução}

A cafeicultura moderna, além de almejar alta produtividade para os produtores, busca desenvolver sistemas de manejo visando a sustentabilidade ambiental da lavoura (PRETTY, 2008), isto é, um sistema agrícola que atenda as necessidades do agricultor e ao mesmo tempo conserve os recursos naturais (CASALINHO et al., 2007). Dentre as atividades realizadas nos sistemas de produção do cafeeiro (Coffea arabica L.), o controle de plantas invasoras é uma operação indispensável, pois se não for realizada, pode ocorrer considerável redução da produtividade (ALCÂNTARA; FERREIRA, 2000a).

As plantas invasoras interferem direta $\mathrm{e}$ indiretamente no sistema de produção do cafeeiro, pois concorrem por água, luz e nutrientes (ALCÂNTARA; FERREIRA, 2000a). Por outro lado, a decomposição de resíduos orgânicos das plantas invasoras libera os nutrientes minerais anteriormente retidos em sua biomassa, de forma gradual e assimilável pelas plantas de cafeeiro, contribuindo para reciclagem dos nutrientes em médio prazo (RICCI; VÍRGILIO FILHO; COSTA, 2008). Os diferentes métodos de controle de plantas invasoras podem alterar significativamente $\mathrm{o}$ solo, seja em suas propriedades físicas, tais como densidade do solo e estabilidade de agregados (ALCÂNTARA; FERREIRA, 2000b; ARAUJOJUNIOR et al., 2011), como nas propriedades químicas (capacidade de troca de cátions, acidez do solo e teor de trocáveis), segundo Alcântara, Nóbrega e Ferreira 2007, e biológicas do solo, (BALOTA; CHAVES, 2011). O controle de plantas invasoras por meio de capinas manuais e herbicidas de pósemergência favorece a manutenção da qualidade física do solo (ALCÂNTARA; FERREIRA, 2000b), uma vez que não ocorre revolvimento intenso do solo e há produção controlada de resíduos acima e abaixo do solo (CARNEIRO et al., 2009). Mesmo assim, quando o manejo de plantas invasoras é realizado com frequência na lavoura cafeeira, é uma das principais causas de compactação do solo, pelo uso excessivo de máquinas agrícolas. Santos et al. (2009) relataram que a utilização de roçadora e herbicida de pré-emergência, associados à enxada rotativa ou grade de discos nas entrelinhas, apresentaram maior tendência à compactação do solo, pois estes implementos são reconhecidamente destruidores da estrutura do solo. Por outro lado, altos índices de cobertura vegetal sobre o solo, seja por plantas invasoras ou não, podem incrementar os teores de matéria orgânica do solo e a atividade microbiológica do solo sob cafezais, devido ao aumento de resíduos orgânicos no solo (MARTINSNETO; MATSUMOTO, 2010).

O carbono orgânico do solo (COS) é um dos principais indicadores de qualidade do solo (CONCEIÇÃO et al., 2005), pois aumenta a disponibilidade de água, atuando ainda como agente cimentante da estrutura, no tampão do $\mathrm{pH}$, na complexação de elementos e capacidade de troca de cátions, especialmente em solos altamente intemperizados do trópico úmido (RODRIGUES; 
GARRIDO, 2005). Atualmente, o COS tem recebido maior atenção por ser um dos principais componentes terrestres do ciclo do $\mathrm{C}$ na biosfera (LAL, 2008). No Brasil, sistemas agrícolas com preparo anual do solo promovem perdas significativas dos estoques de $\operatorname{COS}$ a $0-20 \mathrm{~cm}$ de profundidade, mas isto geralmente não ocorre para as culturas perenes ou sem preparo anual do solo (ZINN; LAL; RESCK, 2005). O cafeeiro, por ser uma cultura perene, somente requer revolvimento intenso do solo durante a implantação da lavoura. Após esta fase, operações de preparo do solo são parciais e periódicas, durante os tratos culturais. A baixa necessidade de aração e gradagem, em relação a culturas anuais, resulta em relativamente baixa destruição de agregados do solo, processo reconhecidamente causador da perda de COS (BERTOL et al., 2000) e da erosão. Ainda, a produção constante de biomassa, típica de culturas perenes, pode favorecer a manutenção ou mesmo o sequestro de COS no cafezal.

Dada a importância da cafeicultura para o Brasil e do COS para a qualidade do solo, é importante avaliar como diferentes práticas de manejo do cafezal o afetam. O objetivo deste estudo foi quantificar os estoques de COS em cafezais sob diferentes métodos de controle de plantas invasoras em um Latossolo do Sul de Minas Gerais, sendo testada a hipótese de que os estoques de COS serão afetados diferentemente pelos métodos empregados.

\section{Material e Métodos}

O estudo foi realizado na Fazenda Experimental da Empresa de Pesquisa Agropecuária do Estado de Minas Gerais (EPAMIG), localizada no município de São Sebastião do Paraíso, MG, situada a 46 55' W 220 255' S. A vegetação original era formada por floresta estacional semidecidual, transicional para Cerrado, com a declividade de média 8\%. O clima da região é classificado como tropical úmido. A temperatura média anual é de $20,8^{\circ} \mathrm{C}$, e a precipitação média anual de $1.470 \mathrm{~mm}$ (ALCÂNTARA;
FERREIRA, 2000b). O solo da área de estudo foi classificado como Latossolo Vermelho distroférrico (EMBRAPA, 2006), argiloso (570 $\mathrm{g} \mathrm{kg}^{-1}$ de argila, $200 \mathrm{~g} \mathrm{~kg}^{-1}$ de areia), desenvolvido sobre basaltos da formação Serra Geral.

A área experimental consiste em lavoura cafeeira implantada em 1974 com a cultivar Catuaí Vermelho IAC-99, no espaçamento 4 x $1 \mathrm{~m}$. Os diferentes sistemas de controle de plantas invasoras (Brachiaria plantaginea Hitch, Digitaria horizontalis Willd, Eleusine indica Gaertn, Bidens pilosa L., Sida spp, Amaranthus viridis L., Portulaca oleracea L., Erigern bonariensis L., Conysa bonariensis L., Emilia sonchifolia DC, Galinsoga parviflora Cav.) foram aplicados nas entrelinhas dos cafeeiros a partir de 1977, sendo conduzidos continuamente até a amostragem. Devido ao declínio de produção com a idade da lavoura, após 30 anos as plantas foram substituídas por outras da cultivar Paraíso, plantadas no espaçamento $4 \times 0,7 \mathrm{~m}$. em janeiro de 2006, sem alteração dos sistemas de controle de invasoras. As recomendações foram seguidas paras as calagem e adubação de acordo com as análises de solo e as recomendações técnicas (CFSEMG COMISSÃO DE FERTILIDADE DE SOLO DE MINAS GERAIS, 1999).

Os tratamentos foram constituídos pelos seguintes métodos: sem capina (SCAP), com plantas invasoras em livre crescimento; capina manual (CAPM), realizada com enxada; roçadora (ROÇA), com largura de corte de 1,3 m; enxada rotativa (ENRT); grade (GRAD) com 1,3 m de largura e na profundidade de $10 \mathrm{~cm}$; herbicida de pósemergência (HPOS), como $0,72 \mathrm{~kg} \mathrm{ha}^{-1}$ glifosato; e herbicida pré-emergente (HPRE), na forma de $0,72 \mathrm{~kg} \mathrm{ha}^{-1}$ de oxyfluorfen. Os tratamentos foram aplicados na parte central das ruas (entrelinhas de plantio), enquanto as laterais das linhas de plantio foram mantidas sempre limpas por capinas manuais ou herbicidas. As operações mecanizadas realizadas para o controle de plantas invasoras passavam a 80 $\mathrm{cm}$ do caule do cafeeiro. O número de operações realizadas durante o ano variou de acordo com o 
método, conforme apresentado na Tabela 1, sendo realizadas sempre que se observava $90 \%$ da rua coberta por plantas invasoras. O delineamento experimental utilizado foi o de blocos casualizados, com três repetições e sete tratamentos, e cada parcela constou de 3 ruas com 154 covas, totalizando 462 covas por tratamento.

Tabela 1. Número médio de operações realizadas por ano, para os métodos de controle de plantas invasoras efetuados para o controle de plantas invasoras no cafeeiro.

\begin{tabular}{lc}
\hline Tratamentos & Operações ano $^{-1}$ \\
\hline Roçadora (ROÇA) & 5 \\
Grade (GRAD) & 3 \\
Enxada rotativa (ENRT) & 3 \\
Herbicida de pós-emergência (HPOS) & 3 \\
Herbicida de pré-emergência (HPRE) & 2 \\
Capina Manual (CAPM) & 5 \\
Testemunha sem capina (SCAP) & - \\
\hline
\end{tabular}

Fonte: Adaptado de Alcântara, Nóbrega e Ferreira (2007).

As amostragens foram realizadas nas entrelinhas de plantio dos cafeeiros a 2,0 $\mathrm{m}$ de distância do caule (EL) e na linha de tráfego a $0,8 \mathrm{~m}$ do caule do cafeeiro (LT), conforme apresentado na Figura 1, nas profundidades de $0-3,10-13$, e $25-28 \mathrm{~cm}$. As amostras indeformadas foram coletadas com amostrador de Uhland (1950), em cilindro métalico, para a medição da densidade do solo (Ds) e propriedades compressivas. As análises de COS e de caracterização do solo foram realizadas em amostras deformadas obtidas nas mesmas camadas. O COS foi determinado pela oxidação úmida em $\mathrm{Na}_{2} \mathrm{Cr}_{2} \mathrm{O}_{7}$ 0,8 $\mathrm{mol}^{1}$ (EMBRAPA, 1997). Os estoques de COS foram calculados como uma média ponderada das duas posições amostradas, e para cada tratamento e posição, nas profundidades 0-10, 10-30 e 0-30 cm, estimando-se a Ds e COS médios para estas por meio de média aritmética simples entre as profundidades amostradas, e pelo método de massa equivalente, o que compensa o efeito da compactação do 'solo. Os estoques de $\mathrm{COS}$ em cafezais como um todo foram calculados assumindo-se que a Ds e teores de COS são os mesmos na linha de plantio do cafeeiro e na entrelinha, conforme verificado por Cogo et al. (2011) em cafezais da mesma região. Os solos da área sob cafezal foram caracterizados quimicamente segundo Embrapa (1997), conforme tabela 2. 
Figura 1. Croquis da parcela experimental do estudo.

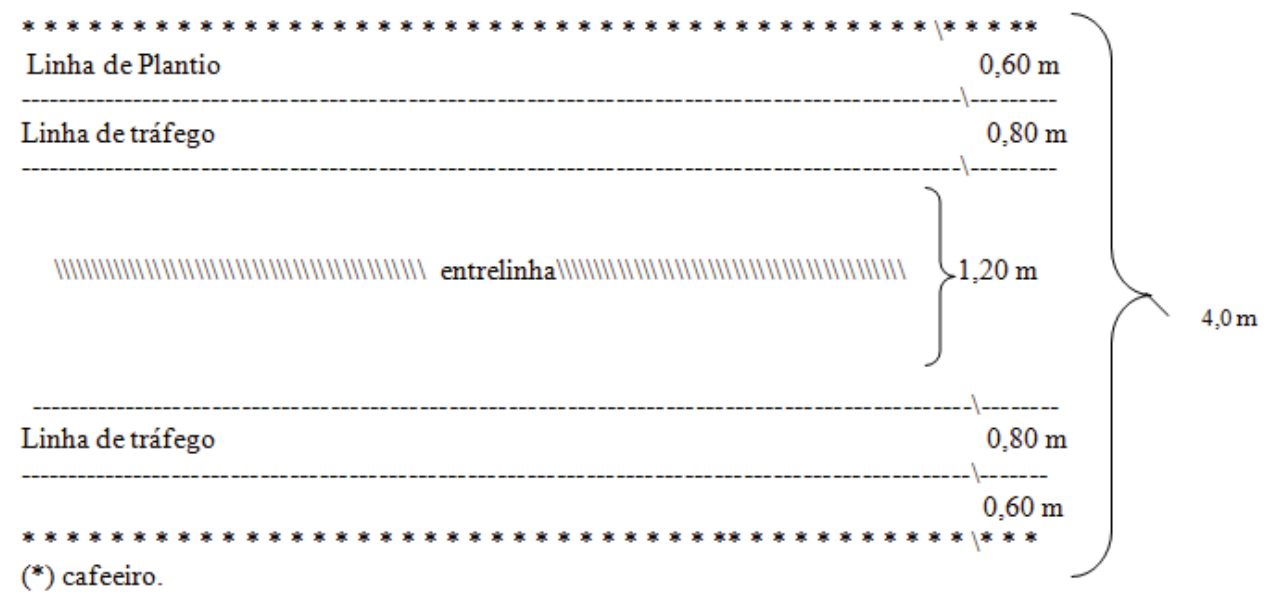

Fonte: Elaboração dos autores.

Tabela 2. Valores médios para os atributos químicos do solo, em função dos diferentes sistemas de manejo de plantas invasoras.

\begin{tabular}{cccccccc}
\hline \multirow{2}{*}{ Trat. } & $\mathrm{pH}$ & $\mathrm{P}$ & $\mathrm{Al}^{3+}$ & $\mathrm{H}+\mathrm{Al}$ & $\mathrm{SB}$ & $\mathrm{T}$ & $\mathrm{V}$ \\
\cline { 2 - 7 } & Água & $\mathrm{mg} / \mathrm{dm}^{-3}$ & $\ldots \ldots \ldots \ldots \ldots \ldots \ldots \ldots \mathrm{cmol}_{c}{ }^{+} / \mathrm{dm}^{3} \ldots \ldots \ldots \ldots \ldots \ldots \ldots \ldots$ & $\ldots \ldots \ldots \ldots \ldots \ldots \ldots \ldots \ldots \ldots \ldots \ldots$ \\
\hline SCAP & 6,70 & 13,5 & 0,10 & 1,90 & 6,70 & 8,63 & 77,9 \\
CAPM & 6,63 & 5,57 & 0,10 & 2,10 & 4,70 & 6,37 & 66,9 \\
HPRE & 5,23 & 1,23 & 0,20 & 4,13 & 1,70 & 5,93 & 31,0 \\
HPOS & 6,43 & 7,13 & 0,10 & 2,60 & 4,50 & 6,87 & 62,0 \\
ENRT & 6,40 & 6,20 & 0,10 & 2,60 & 4,30 & 6,70 & 61,1 \\
ROÇA & 6,43 & 6,17 & 0,00 & 2,50 & 4,10 & 6,73 & 62,8 \\
GRAD & 6,60 & 6,20 & 0,10 & 2,30 & 3,80 & 6,13 & 62,2 \\
\hline
\end{tabular}

${ }^{1}$ SCAP: sem capina; CAPM: capina manual; HPRE: herbicida pré-emergência; HPOS: herbicida pós-emergência; ENRT: enxada rotativa; ROÇA: roçadora; GRAD: grade.

Fonte: Elaboração dos autores.

As análises estatísticas foram realizadas em pacote estatístico JMP 5.1 (SAS, 2003). Para o teste de médias dos tratamentos, foi utilizado o teste de Scott-Knott $(\mathrm{P}<0,05)$, e para verificar diferenças entre as posições de linha de tráfego e entrelinha de plantio utilizou-se o teste $\mathrm{t}(\mathrm{P}<0,05)$.

\section{Resultados e Discussão}

O plantio dos cafezais promoveu o aumento na densidade do solo para a posição entrelinha em relação ao solo sob mata nativa (Tabela 3). De acordo com Araujo-Junior et al. (2011), tal resultado pode ser atribuído aos sistemas de preparo do solo, mecanização em geral e o controle de plantas invasoras que aumentam a densidade do solo, sobretudo na camada $0-3 \mathrm{~cm}$ e $10-13 \mathrm{~cm}$.

Embora o aumento na densidade do solo tenha ocorrido em todos os métodos de controle de plantas invasoras, os valores médios encontrados para as duas posições amostradas foram geralmente menores ao considerado crítico $\left(1,2 \mathrm{~g} \mathrm{~cm}^{-3}\right)$ para degradação estrutural (ARAUJO-JUNIOR et al., 2011), exceto para a profundidade $10-13 \mathrm{~cm}$.

Entre as posições de amostragem não houve em geral diferença na densidade do solo embora, fosse 
esperada maior compactação na linha de tráfego, onde passam as rodas do trator, pois esta é a causa mais frequente de compactação do solo (SANTOS et al., 2009). Este efeito pode ter ocorrido devido à remoção do cafezal plantado em 1974 para implantação da nova lavoura em 2006, o que pode ter obliterado o histórico de tensão da linha de tráfego das máquinas ao longo dos anos (ARAUJO-JUNIOR et al., 2011). Por outro lado, os dados podem também sugerir que o solo sob cafezal, independente da posição, atingiu o máximo de compactação possível para este sistema de preparo e manejo.
Para as profundidades $10-13$ e $25-28 \mathrm{~cm}$, os valores de densidade do solo sob cafezais praticamente não diferem entre si devido ao sistema do controle de plantas invasoras. MartinsNeto e Matsumoto (2010) estudaram sistemas orgânico e convencional de plantio de cafezais, com os respectivos sistemas de controle de plantas invasoras, roçagem e glifosato, e também verificaram que a cobertura vegetal proporcionada pelo acúmulo de resíduos de plantas daninhas pode resultar em menor densidade do solo.

Tabela 3. Densidade do solo (Ds) teor de carbono orgânico do solo (COS) para os diferentes métodos de controle de plantas invasoras e as diferentes posições de amostragem.

\begin{tabular}{|c|c|c|c|c|}
\hline \multirow{2}{*}{$\begin{array}{l}\text { Tratamentos } \\
\text { Posições }\end{array}$} & \multicolumn{2}{|c|}{$\operatorname{COS}\left(\mathrm{g} \mathrm{kg}^{-1}\right)$} & \multicolumn{2}{|c|}{ Ds $\left(\mathrm{g} \cdot \mathrm{cm}^{-3}\right)$} \\
\hline & EL & LT & EL & LT \\
\hline & \multicolumn{4}{|c|}{ 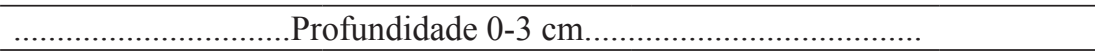 } \\
\hline SCAP $^{1}$ & 2,45 a A & $1,29 \mathrm{~b} \mathrm{~A}$ & 1,02 a DE & 1,12 a B \\
\hline CAPM & 1,51 a BC & 1,41 a $\mathrm{A}$ & 1,19 a $\mathrm{ABC}$ & $1,10 \mathrm{~b} \mathrm{~B}$ \\
\hline HPRE & 1,20 a $\mathrm{C}$ & $0,83 \mathrm{~b} \mathrm{~B}$ & 1,34 a $\mathrm{A}$ & 1,32 a $\mathrm{A}$ \\
\hline HPOS & 1,68 a BC & 1,35 a $\mathrm{A}$ & 1,18 a BC & 1,20 a $\mathrm{AB}$ \\
\hline ENRT & 2,03 a $\mathrm{AB}$ & $1,41 \mathrm{~b} \mathrm{~A}$ & 1,11 a $\mathrm{CD}$ & 1,10 a $\mathrm{AB}$ \\
\hline ROÇA & $2,21 \mathrm{a} \mathrm{AB}$ & $1,53 \mathrm{~b} \mathrm{~A}$ & 1,20 a $\mathrm{ABC}$ & $1,24 \mathrm{a} A B$ \\
\hline GRAD & 1,85 a $\mathrm{ABC}$ & $1,25 \mathrm{~b} \mathrm{~A}$ & 1,29 a $\mathrm{AB}$ & 1,25 a B \\
\hline \multirow[t]{2}{*}{ MATA } & $1,57 \mathrm{BC}$ & & $0,89 \mathrm{E}$ & \\
\hline & \multicolumn{4}{|c|}{ 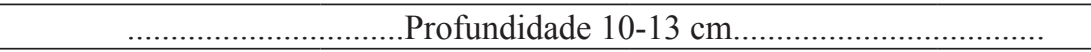 } \\
\hline $\mathrm{SCAP}^{1}$ & 1,43 a $\mathrm{A}$ & $0,90 \mathrm{~b} \mathrm{~B}$ & 1,22 a $\mathrm{A}$ & 1,26 a $\mathrm{A}$ \\
\hline CAPM & 1,10 a BC & $0,85 \mathrm{~b} \mathrm{~B}$ & 1,26 a $\mathrm{A}$ & 1,22 a A \\
\hline HPRE & 0,92 a C & $0,68 \mathrm{~b} \mathrm{C}$ & 1,30 a $\mathrm{A}$ & 1,26 a A \\
\hline HPOS & 1,14 a BC & $0,96 \mathrm{~b} \mathrm{~B}$ & 1,26 a A & 1,26 a A \\
\hline ENRT & 1,00 a $\mathrm{C}$ & 0,93 a B & 1,24 a $\mathrm{A}$ & 1,23 a $\mathrm{A}$ \\
\hline ROÇA & 1,27 a $\mathrm{AB}$ & 1,08 a $\mathrm{A}$ & $1,23 \mathrm{~b} \mathrm{~A}$ & 1,28 a $\mathrm{A}$ \\
\hline GRAD & 1,00 a C & 0,87 a $\mathrm{B}$ & 1,23 a $\mathrm{A}$ & 1,25 a A \\
\hline \multirow[t]{2}{*}{ MATA } & $1,28 \mathrm{AB}$ & & $0,98 \mathrm{~B}$ & \\
\hline & \multicolumn{4}{|c|}{ 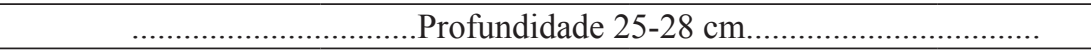 } \\
\hline SCAP $^{1}$ & 0,98 a $\mathrm{AB}$ & $0,75 \mathrm{~b} \mathrm{AB}$ & 1,20 a $\mathrm{A}$ & 1,16 a C \\
\hline CAPM & 0,83 a $\mathrm{AB}$ & 0,68 a $\mathrm{AB}$ & 1,17 a $\mathrm{A}$ & 1,17 a C \\
\hline HPRE & 0,73 a $\mathrm{B}$ & 0,63 a $\mathrm{B}$ & 1,17 a $A$ & $1,14 \mathrm{a} A B$ \\
\hline HPOS & 0,83 a $\mathrm{AB}$ & $0,69 \mathrm{a} A B$ & $1,22 \mathrm{a} A B$ & $1,17 \mathrm{a} A \mathrm{~B}$ \\
\hline ENRT & $0,85 \mathrm{a} A \mathrm{~B}$ & $0,73 \mathrm{a} A B$ & $1,22 \mathrm{a} A B$ & 1,15 a C \\
\hline ROÇA & $0,87 \mathrm{a} A B$ & 0,77 b A & $1,19 \mathrm{~b} \mathrm{~A}$ & $1,24 \mathrm{a} \mathrm{A}$ \\
\hline GRAD & $0,94 \mathrm{a} \mathrm{AB}$ & $0,75 \mathrm{a} A B$ & 1,18 a $\mathrm{A}$ & 1,18 a BC \\
\hline MATA & $1,04 \mathrm{~A}$ & & $1,09 \mathrm{~B}$ & \\
\hline
\end{tabular}

Na mesma profundidade, médias seguidas pela mesma letra maiúscula nas colunas e minúsculas nas linhas para o mesmo atributo nas diferentes posições de amostragem não diferem entre si pelo teste de Tukey a 5\% de probabilidade. ${ }^{1}$ SCAP: sem capina; CAPM: capina manual; HPRE: herbicida pré-emergência; HPOS: herbicida pós-emergência; ENRT: enxada rotativa; ROÇA: roçadora; GRAD: grade; MATA: mata nativa; LT: linha de tráfego; EL: centro das entrelinhas dos cafeeiros.

Fonte: Elaboração dos autores. 
Os teores de COS diminuem com a profundidade em todos os solos, devido ao maior aporte de matéria orgânica na superfície. Verifica-se que o teor de COS na entrelinha para todos os tratamentos apresenta valores próximos ao da mata nativa e em alguns casos maior (sem capina a 0-3 cm), conforme apresentado na Tabela 3 . Isto pode ser explicado pela alta densidade de plantas invasoras, e consequente produção de biomassa vegetal. Martins-Neto e Matsumoto (2010) relatam que o volume de cobertura vegetal acumulado no solo pode influenciar os teores de matéria orgânica do solo, favorecendo a ação dos microorganismos e a humificação dos resíduos produzidos.

O maior decréscimo no teor de COS em relação à mata foi verificado para o manejo herbicida de pré-emergência em todas as camadas amostradas (Tabela 3), o que pode ser resultado da ausência de biomassa na entrelinha por 3 décadas. Os teores de COS nas profundidades $0-3$ e $10-13 \mathrm{~cm}$ em todos os cafezais seguem a tendência a ser menores na linha de tráfego do que na entrelinha, pois o acesso constante dos tratores nos diversos tratos culturais diminui ou elimina a emergência das plantas, reduzindo o acúmulo de seus resíduos orgânicos. Para a profundidade $25-28 \mathrm{~cm}$, isto ocorreu apenas sob os manejos sem capina e roçadora.

Houve diferença significativa entre os tratamentos e para as camadas $0-10,10-30$ e 0-30 $\mathrm{cm}$, para os estoques de COS, apresentados na Figura 2. O tratamento herbicida em pré-emergência apresenta os menores estoques de COS, visto que se caracteriza por reduzir ao máximo a vegetação do solo, causando uma perda de aproximadamente $35 \%$ do estoque inicial.

Figura 2. Estoque de carbono orgânico no solo em função dos sistemas de manejo de plantas invasoras.

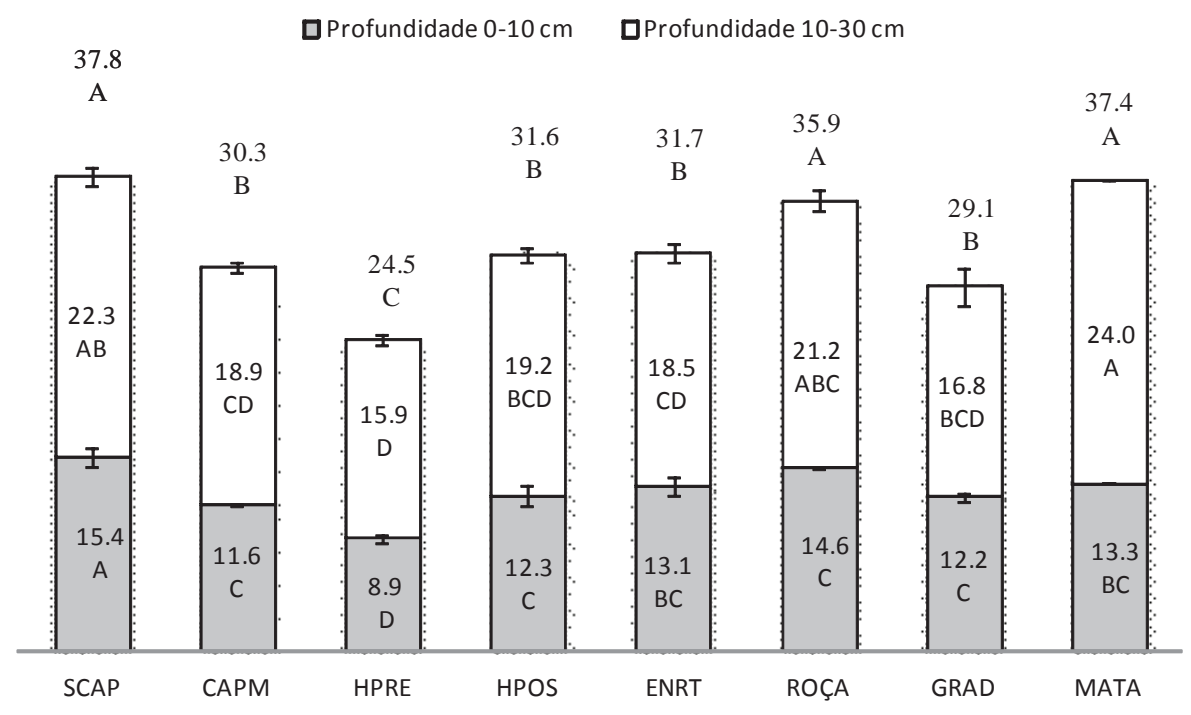

Obs.: As médias seguidas da mesma letra na mesma profundidade não diferem entre si pelo teste de Tukey a $5 \%$ de probabilidade.

Fonte: Elaboração dos autores. 
Os tratamentos em que se aplicou herbicida de pós-emergência, capina manual, enxada rotativa e grade mostraram redução dos estoques de COS em torno de $20 \%$. Este resultado pode ser atribuído à baixa biomassa das invasoras ou à destruição da estrutura do solo, facilitando a oxidação do COS. Os tratamentos sem capina e roçadora apresentaram estoques de COS iguais ao da mata referência. Este efeito sugere que o manejo das plantas invasoras não compromete necessariamente os estoques de $\operatorname{COS}$ em cafezais convencionais, o que merece ser melhor investigado, devido à importância da agricultura no ciclo do C no sistema solo-atmosfera,

\section{Conclusões}

1. Houve pouca diferença em teores de COS entre a mata nativa e posição entrelinha dos cafeeiros;

2. Os tratamentos sem capina e roçadora apresentam estoque de COS semelhantes ao da mata nativa.

3. Os tratamentos causaram perdas de $20 \%$ do estoque de $\operatorname{COS}$ a 0-30 cm, enquanto o herbicida pré-emergência causou a redução de $35 \%$, em relação à mata nativa.

\section{Agradecimentos}

Os autores agradecem ao Consórcio Brasileiro de Pesquisa e Desenvolvimento do Café (CBP\&D/ Café) pelo apoio financeiro para o desenvolvimento do presente estudo. Aos funcionários da Fazenda Experimental da EPAMIG em São Sebastião do Paraíso - MG, especialmente a Paulo G.de Azevedo, Homero J. Lemos, Antônio E. Pereira Xavier e Juracy Júnior de Oliveira, os quais contribuíram para a realização deste trabalho. À FAPEMIG (Projeto CAG-APQ/01352-09) pelo apoio financeiro.

\section{Referências}

ALCÂNTARA, E. N.; FERREIRA, M. M. Efeito de diferentes métodos de controle de plantas daninhas sobre a produção de cafeeiros instalados em Latossolo Roxo Distrófico. Ciência e Agrotecnologia, Lavras, v. 24, n. 1, p. 54-61, 2000a.

Efeito de métodos de controle de plantas daninhas na cultura do cafeeiro (Coffea arabica L.) sobre a qualidade física do solo. Revista Brasileira de Ciência do Solo, Viçosa, v. 24, n. 4, p. 711-721, 2000 b.

ALCÂNTARA, E. N.; NÓBREGA, J. C. A.; FERREIRA, M. M. Métodos de controle de plantas invasoras na cultura do cafeeiro (Coffea arabica L.) e componentes da acidez do solo. Revista Brasileira de Ciência do Solo, Viçosa, v. 31, n. 1, p. 1525-1533, 2007.

ARAUJO-JUNIOR, C. F.; DIAS JUNIOR, M. S.; GUIMARÃES, P. T. G.; ALCÂNTARA, E. N. Capacidade de suporte de carga e umidade crítica de um Latossolo induzida por diferentes manejos. Revista Brasileira de Ciência do Solo, Viçosa, v. 35, n. 1, p. 115-131, 2011.

BAlOTA, E. L.; CHAVES, J. C. D. Enzymatic activity and mineralization of carbon and nitrogen in soil cultivated with coffee and green manures. Revista Brasileira de Ciência do Solo, Viçosa, v. 34, n. 5, p. 115131, 2011.

BERTOL, I.; SCHICK, J.; MASSARIOL, J. M.; REIS, E.; DILY, L. Propriedades físicas de um cambissolo húmico álico afetadas pelo manejo do solo. Ciência Rural, Santa Maria, v. 30, n. 1, p. 91-95, 2000.

CARNEIRO, C. E. A.; MELÉM JÚNIOR, N. J.; AZEVEDO. M. C. B.; ANDRADE, E. A. Efeitos dos sistemas de manejo sobre o carbono orgânico total e carbono residual de um latossolo vermelho eutroférrico. Semina: Ciências Agrárias, Londrina, v. 30, n. 1, p. 5-10, 2009.

CASALINHO, H. D.; MARTINS, S. R.; SILVA, J. B.; LOPES, A. S. Qualidade do solo como indicador de sustentabilidade de agroecossistemas. Revista Brasileira de Agrociência, Pelotas, v. 13, n. 2, p. 195-203, 2007.

COGO, F. D.; GUERRA, R. A.; CUNHA, R. L.; ZINN, Y. L. Propriedades do solo em cafezais e suas sucessões no sul de Minas Gerais. In: SIMPÓSIO DE PESQUISA DOS CAFÉS DO BRASIL, 7., 2011, Araxá, MG. Anais... Brasília, D. F: Embrapa-Café, 2011. 1 CD-ROM.

COMISSÃO DE FERTILIZANTES DO SOLO DO ESTADO DE MINAS GERAIS - CFSEMG. Recomendações para o uso de corretivos e fertilizantes em Minas Gerais, $5^{a}$ aproximação. Viçosa: UFV, 1999. $322 \mathrm{p}$. 
CONCEIÇÃO, P. C.; AMADO, T. J. C.; MIELNICZUK, J.; SPAGNOLLO, E. Qualidade do solo em sistemas de manejo avaliada pela dinâmica da matéria orgânica e atributos relacionados. Revista Brasileira de Ciência do Solo, Viçosa, v. 29, n. 5, p.777-788, 2005.

EMPRESA BRASILEIRA DE PESQUISA AGROPECUÁRIA - EMBRAPA. Centro Nacional de Pesquisa de Solos. Manual de métodos de análise de solo. 2. ed. Brasília: Embrapa, 1997. 212 p.

. Sistema brasileiro de classificação de solos. 2. ed. Brasília: Embrapa, 2006. 306 p.

LAL, R. Sequestration of atmospheric CO2 in global carbon pools. Energy and Environmental Science, London, v. 1, n. 1, p. 86-100, 2008.

MARTINS-NETO, F. L.; MATSUMOTO, S. N.; Qualidade do solo e nutrição de plantas em sistemas de produção de café (Coffea arabica L.). Coffee Science, Lavras, v. 5, n. 3, p. 206-213, 2010.

PRETTY, J. Agricultural sustainability: concepts, principles and evidence. Philosophical Transactions of the Royal Society B-Biological Sciences, London, v. 363, n. 1491, p. 447-465, 2008.
RICCI, M. S. F.; VÍRGILIO FILHO, E. M.; COSTA, J. R. Diversidade da comunidade de plantas invasoras em sistemas agroflorestais com café em Turrialba, Costa Rica. Pesquisa Agropecuária Brasileira, Brasília, v. 43, n. 7, p. 825-834, 2008.

RODRIGUES, F. S.; GARRIDO, R. G. Fluxo sazonal de $\mathrm{NO}_{3}$ no trópico úmido. Revista Científica Eletrônica de Agronomia, Garça, v. 4, n. 8, p. 1-9, 2005.

SANTOS, G. A.; DIAS-JUNIOR, M. S. D.; GUIMARÃES, P. T. G.; ARAÚJO-JUNIOR, C. C. F. Capacidade de suporte de carga de um latossolo influenciada pelo manejo de plantas invasoras em lavoura cafeeiras. Coffee Science, Lavras, v. 4, n. 2, p. 165-177, 2009.

SAS Institute 2003. JMP, statistics and graphics guide, Version 5.1, SAS Inc., Cary, NC, 593 p.

UHLAND, R. E. Physical properties of soils as modified by crops and management. Soil Science Society of America Proceedings, Madison, v. 14, n. C, p. 361-366, aug. 1950.

ZINN, Y. L.; LAL, R.; RESCK, D. V. S. Changes in soil organic carbon stocks under agriculture in Brazil. Soil \& Tillage Research, Amsterdam, v. 84, n. 1, p. 28-40, 2005. 
